
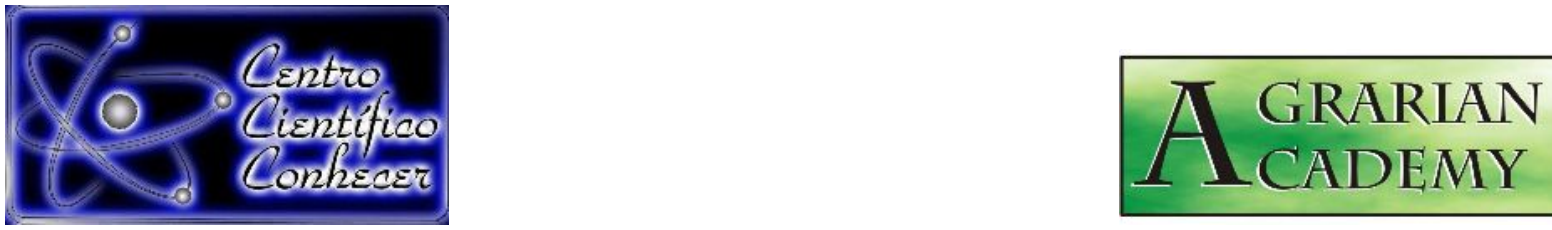

\title{
MESOFAUNA EDÁFICA NA MACAXEIRA, CAPIM COLONIÃO E EM FRAGMENTO DE MATA ATLÂNTICA
}

\author{
Demichaelmax Sales de Melo1; José Carlos da Costa2; Antonio Alves Pinto; Felipe \\ Thomaz da Camara ${ }^{4}$; Ytalo Roberto Pereira Damaceno ${ }^{1}$ \\ 1 Mestrando em Produção Vegetal pela Universidade Federal Rural do Pernambuco/ \\ UFRPE, Garanhuns-PE \\ 2 Professor Adjunto do Instituto Federal de Pernambuco/IFPE, Vitória do Santo Antão-PE \\ ${ }^{3}$ Graduando em Agronomia pela Universidade Federal do Cariri/UFCA, Crato-CE \\ (antonioufca@gmail.com) \\ ${ }^{4}$ Professor Adjunto da UFCA, Crato-CE
}

Recebido em: 14/07/2018 - Aprovado em: 28/07/2018 - Publicado em: 31/07/2018 DOI: 10.18677/Agrarian_Academy_2018a38

\begin{abstract}
RESUMO
Devido à sensibilidade que a fauna edáfica possui ao tipo de manejo do solo, tem-se utilizado esses organismos como bioindicadores para avaliar a degradação do solo. O presente trabalho teve como objetivo estimar a densidade da mesofauna edáfica na macaxeira (Manihot esculenta), capim colonião (Panicum maximum) e fragmento florestal de Mata Atlântica no Instituto Federal do Pernambuco, Vitória de Santo Antão-PE. O experimento foi conduzido em delineamento inteiramente casualizado, em arranjo fatorial $3 \times 2$ com quatro repetições totalizado 24 observações. O primeiro fator foi constituído por três áreas com diferentes usos (fragmento florestal de Mata Atlântica, área cultivada com macaxeira e outra com capim colonião), e o segundo fator de duas épocas de coleta (estação seca e chuvosa). Em cada área foram coletadas oito amostras nas estações seca e chuvosa, com auxílio de anéis metálicos de aproximadamente $7 \mathrm{~cm}$ de altura e $6 \mathrm{~cm}$ de diâmetro, na profundidade de zero a $10 \mathrm{~cm}$. As amostras foram levadas para os Funis de Berlese e análises químicas e físicas. A partir das amostras provenientes colocadas nos Funis de Berlese foi possível mensurar os indivíduos da mesofauna (táxon). Foram determinados os Índices de Constância e Índice de Diversidade de Shannon $(H)$. Os diferentes ambientes influenciaram na ocorrência das classes da mesofauna. A classe Arachnida apresentou maior frequência no ambiente de mata atlântica que as demais classes: Entognatha, Insecta e Symphyla, no entanto não tiveram diferença significativa. Na cultura da macaxeira foi encontrada a maior desuniformidade em relação aos organismos edáficos.
\end{abstract}

PALAVRAS-CHAVE: Ambientes, Diversidade; Organismos edáficos; Solo;

\section{EDAPHIC MESOFAUNA IN CASSAVA, PANICUM MAXIMUM, AND ATLANTIC FOREST FRAGMENTS}

\footnotetext{
ABSTRACT

Due to the sensibility that the edaphic fauna has to the type of soil management, these organisms have been used as bioindicators to evaluate soil degradation. This AGRARIAN ACADEMY, Centro Científico Conhecer - Goiânia, v.5, n.9; p. 3902018
} 
study aimed to evaluate the density of the soil fauna in the macaxeira (manihot esculenta), panicum maximum and atlantic forest fragments at the instituto federal de educação, ciência e tecnologia de pernambuco (IFPE) - campusvitória, in the municipality of vitória de santo antão-PE. in each area, 8 samples were collected in the dry and rainy seasons, using metal rings of approximately $7 \mathrm{~cm}$ in height and $6 \mathrm{~cm}$ per sample, at a depth of $0-10 \mathrm{~cm}$. samples of soil were conditioned in plastic bags, to minimize losses of moisture, immediately after the collection, the samples were taken to the funles de berlese. soil samples for chemical and physical analyzes were performed systematically. for each group of the fauna soil's taxon and their respective constancy index was determined and in the evaluation of the ecological behavior of the mesofauna, the total number of individuals was determined. the comparisons were made between the areas using the shannon diversity index (h). the different environments influenced the occurrence of classes. arachnida class presented higher frequency in the forest environment and the other classes entognath, insect and symphylan however did not have significant difference between them. the forest environment presented better conditions for the soil organisms. in the culture of the manioc was found the greater unevenness in relation to the edaphic organisms.

KEYWORDS: density; edaphic organisms; soil; environments

\section{INTRODUÇÃO}

O termo solo origina-se do Latim solum e significa suporte, superfície, base. No entanto ao observar o solo de forma sutil, a impressão é que não existe vida neste conjunto de partículas. Porém, se observar com delicadeza, uma grande e diversificada população de organismos vivos habitam o solo e exercem papeis importantes (SILVA et al., 2012).

$\mathrm{Na}$ agricultura, o solo não possui apenas função de sustentação para as culturas nele implantadas, tem também a capacidade de fornecer água e íons minerais. Além disso, faz parte do nicho ecológico onde se encontram os organismos edáficos responsáveis pela decomposição da matéria orgânica, produção de húmus, ciclagem de nutrientes e a produção de complexos que causam a agregação do solo (BERUDE et al., 2015).

No solo a fauna pode ser classificada de acordo com o diâmetro corporal em microfauna, mesofauna e macrofauna (MACHADO et al., 2015). A mesofauna abrange os organismos entre 0,2 a 2,0 mm, incluindo, por exemplo, ácaros, colêmbolos e alguns insetos. As atividades tróficas desses seres incluem tanto o consumo de microrganismos como a fragmentação de material vegetal em decomposição (BERUDE et al., 2015), desse modo, a variação do habitat natural desses organismos determina quais os grupos da fauna permanecerão no ambiente modificado.

Com base na sensibilidade que a fauna edáfica possui ao uso de diferentes coberturas vegetais e de práticas culturais, tem-se utilizado esses organismos como bioindicadores para avaliar a degradação do solo (NUNES et al., 2012). Tendo em vista que varia conforme as características do habitat, clima, tipo de solo e quantidade de matéria orgânica (MACHADO et al., 2015).

Trabalhos sobre a influência da cobertura vegetal e manejo sobre a mesofauna edáfica na zona da mata de Pernambuco ainda são escassos. Nessa perspectiva pretendeu-se mensurar como o impacto das formas de utilização do solo sobre a mesofauna edáfica pode fornecer dados para mitigar o efeito da ação 
antrópica no ambiente solo. Assim, o presente trabalho teve como objetivo estimar a densidade e a diversidade da mesofauna edáfica em diferentes sistemas de uso do solo.

\section{MATERIAIS E MÉTODOS}

O estudo foi realizado no Instituto Federal de Educação, Ciência e Tecnologia de Pernambuco (IFPE) - Campus Vitória, no município Vitória de Santo Antão, PE (080'59" S e 3517'25" W) com predominância de Argissolo Vermelhoamarelo (ARAÚJO FILHO et al., 2000), com altitude média de $155 \mathrm{~m}$. O clima da região é correspondente à classificação AS de Köppen (ALVARES et al., 2013), clima tropical com estação seca no período em que o Sol está mais alto (está no mesmo hemisfério) e os dias são mais longos, com temperatura média de $24,1^{\circ} \mathrm{C}$ e pluviosidade média anual de $1.014 \mathrm{~mm}$.

O experimento foi conduzido em delineamento inteiramente casualizado, em arranjo fatorial $3 \times 2$, com quatro repetições. O primeiro fator foi constituído por três áreas com diferentes usos (fragmento florestal de Mata Atlântica, área cultivada com macaxeira e outra com capim colonião), e o segundo fator de duas épocas de coleta (estação seca e chuvosa).

Em cada sistema de uso do solo foram demarcadas quatro parcelas (Repetições) de aproximadamente $5 \times 5 \mathrm{~m}\left(25 \mathrm{~m}^{2}\right)$ totalizando $100 \mathrm{~m}^{2}$, seguindo a declividade do terreno. Nesta área demarcada foram coletadas no mês de julho de 2017 amostras de solo deformadas, com trado holandês, para a análise química, que foram acondicionadas em sacos plásticos para posterior análise em laboratório. Para cada área foram realizadas três amostras simples para cada tratamento formando uma amostra composta por área.

A profundidade de coleta foi de 0 a $10 \mathrm{~cm}$. As análises químicas foram efetuadas no Laboratório de Química e Fertilidade dos solos do IFPE - Campus Vitória de Santo Antão. Em relação aos parâmetros físicos do solo foram retiradas três amostras de solo indeformadas, sendo analisada a densidade do solo, a porosidade e a temperatura do solo.

A densidade do solo foi realizada pelo método do anel volumétrico, conforme descrito por Donagema et al. (2011) e para a porosidade total utilizaram-se os índices de densidade do solo e densidade de partículas no cálculo, de acordo com metodologia descrita por Donagema et al. (2011). Para a temperatura do solo foram mensurados três valores por área, utilizando-se um termômetro digital para solos e feita uma média para cada tratamento.

Para a análise da mesofauna foram retiradas oito amostras em cada área, nas estações seca e chuvosa, com auxílio de anéis metálicos de aproximadamente $7 \mathrm{~cm}$ de altura e $6 \mathrm{~cm}$ de diâmetro, totalizando $197,92 \mathrm{~cm}^{3}$ de volume de solo por amostra, na profundidade de 0 a $8 \mathrm{~cm}$.

As amostras de solo foram acondicionadas em sacos plásticos, visando minimizar perdas da umidade do material. Imediatamente após a coleta, as amostras foram levadas para as mesas extratoras onde estavam instalados os Funis de Berlese, conforme Scoriza et al. (2016). As amostras permaneceram sete dias em Baterias extratoras de Berlese, cuja fonte de calor e luz foram lâmpadas incandescentes de $25 \mathrm{~W}$. Os organismos foram coletados em frascos de vidro com líquido conservante de álcool $70 \%$.

Passado o período de extração nas mesas, os frascos foram etiquetados e posteriormente o material contido em cada frasco foi transferido para placas de Petri. 
A triagem, contagem e identificação dos diferentes grupos da mesofauna foram realizadas com o auxílio de um microscópio estereoscópico (Tecnival modelo SQFF) no IFPE - Campus Vitória de Santo Antão.

Para cada grupo da mesofauna (táxon) foi determinado o respectivo Índice de Constância durante o período de coletas nas três áreas estudadas. A Constância refere-se à relação entre o número de coletas da espécie estudada e o total de coletas (RIBEIRO et al., 2016):

$$
\mathrm{C}(\%)=\mathrm{P} / \mathrm{N} \times 100
$$

Onde: $\mathrm{C}=$ Constância, $\mathrm{P}=$ Número de coletas em que constou $\mathrm{O}$ táxon estudado, e $\mathrm{N}$ = Número total de coletas efetuadas.

Obtidas as porcentagens, as espécies foram agrupadas em categorias, de acordo com a classificação de Aguiar e Gaglianone (2012) em Taxas constantes (presentes em mais de $50 \%$ das coletas), Taxas acessórios (presentes entre 25 e $50 \%$ das coletas) e Taxas acidentais (presentes em menos de $25 \%$ das coletas).

A riqueza foi determinada pelo número de grupos taxonômicos encontrados e a abundância pelo número total de indivíduos de cada táxon. $\mathrm{Na}$ avaliação do comportamento ecológico da mesofauna, foi determinada a quantidade total de indivíduos e foram realizadas comparações entre as áreas utilizando o Índice de Diversidade de Shannon $(H)$, definido por:

$\mathrm{H}=-\Sigma \mathrm{pi} . \log \mathrm{pi}$,

Onde: $\mathrm{pi}=\mathrm{ni} / \mathrm{N} ; \mathrm{ni}=$ densidade de cada grupo; $\mathrm{N}=\Sigma$ da densidade de todos os grupos (BEGON et al., 1996).

Os dados foram submetidos à análise de variância, e quando observado efeito significativo ao teste de Tukey a $5 \%$ de probabilidade.

\section{RESULTADOS E DISCUSSÕES}

Na tabela 1 estão apresentados os resultados dos parâmetros químicos dos solos analisados, possibilitando a comparação da fertilidade do solo nas três áreas estudadas.

TABELA 1. Análise química dos solos das áreas estudadas, Vitória de Santo Antão 2017.

\begin{tabular}{|c|c|c|c|}
\hline \multirow{2}{*}{ Analises } & \multicolumn{3}{|c|}{ Ambientes } \\
\hline & Macaxeira & Mata atlântica & Capim colonião \\
\hline $\mathrm{pH}(\mathrm{H} 2 \mathrm{O})$ & 5,6 & 6,1 & 5 \\
\hline & & ---cmolc/dm3-- & ----------- \\
\hline $\mathrm{Ca} 2+$ & 1,3 & 1,8 & 1,1 \\
\hline $\mathrm{Mg} 2$ & 1,5 & 1,5 & 0,8 \\
\hline $\mathrm{K}+$ & 0,2 & 0,4 & 0,2 \\
\hline $\mathrm{Na}+$ & 0,2 & 0,2 & 0,2 \\
\hline SB & 3,1 & 3,8 & 2,2 \\
\hline $\mathrm{Al} 3+$ & 0,3 & 0,2 & 0,3 \\
\hline CTCe & 3,4 & 4,1 & 2,5 \\
\hline $\mathrm{H}+\mathrm{Al}$ & 4,2 & 3,6 & 4,4 \\
\hline \multirow[t]{2}{*}{$\mathrm{T}$} & 7,3 & 7,5 & 6,6 \\
\hline & \multicolumn{3}{|l|}{--} \\
\hline $\mathrm{V}$ & 42,7 & 51,3 & 33,5 \\
\hline \multirow[t]{2}{*}{ M } & 8,3 & 5,4 & 11,3 \\
\hline & \\
\hline$P$ & 56,3 & 24,7 & 8,7 \\
\hline
\end{tabular}

AGRARIAN ACADEMY, Centro Científico Conhecer - Goiânia, v.5, n.9; p.393 2018 
De acordo com a análise química do solo (Tabela 1) a mata atlântica obteve o maior índice de $\mathrm{pH}$ e os maiores valores observados para a concentração de cálcio $\left(\mathrm{Ca}^{2+}\right)$ e potássio $\left(\mathrm{K}^{+}\right)$além de apresentar maiores valores para a capacidade de troca de cátions a $\mathrm{pH} 7,0$ (T) e porcentagem de saturação por bases (V\%). Enquanto que a macaxeira obteve maior valor da concentração do elemento Fósforo (P) e manteve valor igual ao da concentração de Magnésio do primeiro tratamento e os mesmos valores das concentrações de Alumínio $\left(\mathrm{Al}^{3^{+}}\right)$e Sódio $\left(\mathrm{Na}^{+}\right)$da terceira área.

Tais resultados podem ter sofrido influência dos invertebrados do solo, pois alteram as populações e a atividade dos microrganismos responsáveis pelos processos de mineralização e humificação da matéria orgânica do solo e, portanto, exercem influência sobre a disponibilidade de nutrientes assimiláveis pelas plantas (BERUDE et al., 2016).

$\mathrm{Na}$ tabela 2, verifica-se que para os índices de densidade do solo foi menor na mata atlântica, seguida das áreas de capim e cultivo de macaxeira. Tais resultados podem estar relacionados às práticas de preparo do solo que contribuem com a compactação do solo e consequentemente o aumento da densidade do solo (CALONEGO et al., 2012).

TABELA 2. Atributos físicos dos solos das áreas estudadas.

\begin{tabular}{ccccc}
\hline & Densidade do solo & Porosidade & \multicolumn{2}{c}{ Temperatura do solo ${ }^{\circ} \mathbf{C}$} \\
\cline { 3 - 5 } Ambientes & $\mathrm{g} / \mathrm{cm}^{3}$ & $\%$ & Chuvoso & Seco \\
\cline { 2 - 5 } Macaxeira & 1,00 & 37,07 & 23,5 & 27,5 \\
Mata atlântica & 0,94 & 44,18 & 22,0 & 23,5 \\
Capim colonião & 0,97 & 41,26 & 24,0 & 25,5 \\
\hline
\end{tabular}

Em relação à porosidade (Tabela 2) a mata atlântica obteve a maior porcentagem de porosidade, seguido do capim colonião e posteriormente a macaxeira. Esses resultados devem-se ao aumento da matéria orgânica, que eleva a quantidade de poros e diminui a densidade do solo, refletindo em melhorias na estrutura (OLIVEIRA et al., 2015).

Para a temperatura média do solo durante a estação chuvosa (Tabela 2) o maior valor foi observado no capim colonião. Já na estação seca a maior temperatura foi observada na macaxeira. Verifica-se ainda na Tabela 2 que o ambiente mata atlântica obteve as menores temperaturas nas duas estações, tais resultados podem estar relacionados ao acúmulo da matéria orgânica, conforme Costa et al. (2013), a matéria orgânica auxilia na estabilização da temperatura do solo, favorecendo o aumento da capacidade de retenção de água no solo, o desenvolvimento das raízes, além de aumentar a fauna do solo. Com relação à mesofauna do solo, foram identificadas ao todo quatro classes (Figura 2), Arachnida, Entognatha, Insecta e Symphyla. 


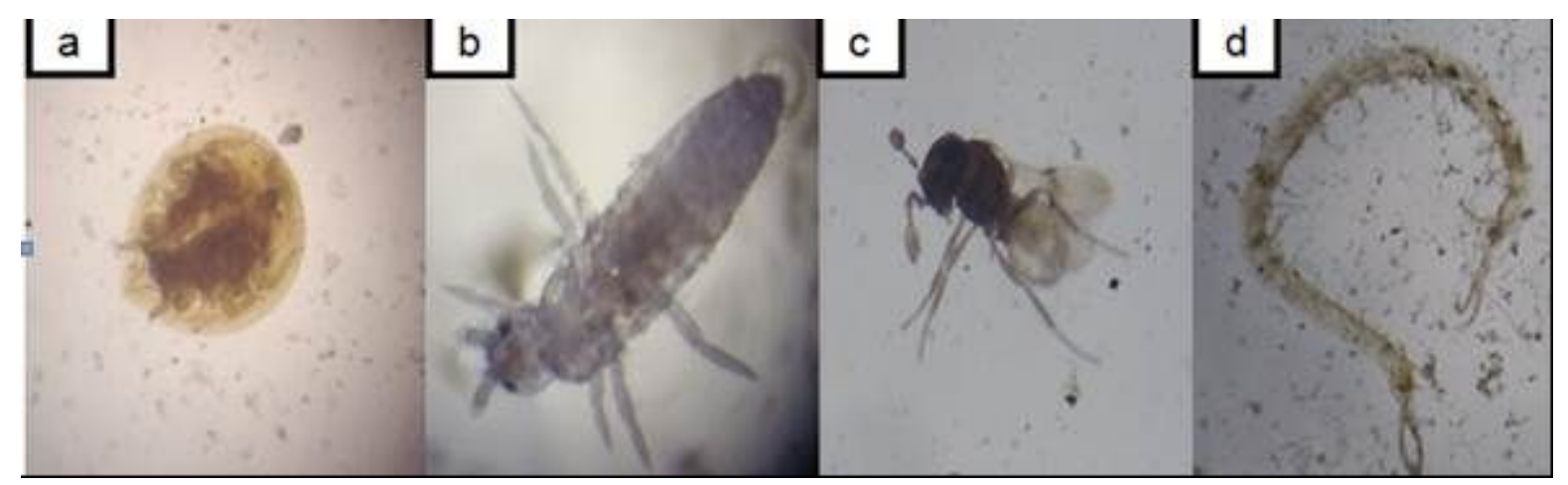

FIGURA 1: Grupos da mesofauna encontrados nas amostras Arachnida (a), Entognatha (b), Insecta (c) e Symphyla (d). Vitória de Santo Antão, PE $-2017$.

Fonte: autores(2017).

O nível de ocorrência de cada grupo da mesofauna, e a classificação destes índices, encontram-se na Tabela 3.

TABELA 3. Distribuição dos índices de constância para os diferentes grupos da mesofauna, coletados nas três áreas estudadas na estação seca e chuvosa.

\begin{tabular}{ccccccccc}
\hline & \multicolumn{2}{c}{ Arachnida } & \multicolumn{2}{c}{ Entognatha } & \multicolumn{2}{c}{ Insecta } & \multicolumn{2}{c}{ Symphyla } \\
\cline { 2 - 9 } & Chuvosa & Seca & Chuvosa & Seca & Chuvosa & Seca & Chuvosa & Seca \\
\hline Ambientes & & & & & & & & \\
Macaxeira & 12,5 & 12,5 & 0 & 0 & 37,5 & 37,5 & 12,5 & 0 \\
Mata Atlântica & 15,0 & 100,0 & 12,5 & 25,0 & 25,0 & 37,5 & 12,5 & 25,0 \\
Cap. colonião & 12,5 & 25,0 & 37,5 & 0 & 12,5 & 37,5 & 0 & 25,0 \\
\hline
\end{tabular}

Constantes - presentes em mais de $50 \%$ das amostras; Acessórios - presentes entre $25 \mathrm{e}$ $50 \%$ das amostras; Acidentais - presentes em menos de $25 \%$ das amostras (AGUIAR e GAGLIANONE, 2012).

$\mathrm{Na}$ tabela 3, verifica-se que na estação chuvosa, as classes Arachnida e Symphyla obtiveram taxas acidentais para os três ambientes, enquanto que para a classe Entognatha e Insecta a maior parte dos tratamentos foram considerados taxas acessórios.

$\mathrm{Na}$ estação seca o tratamento com macaxeira apresentou taxa acidental, para as classes Arachnida, Entognatha e symphyla, já para o tratamento mata atlântica e capim colonião houve predominância da taxa acessória, com a mata atlântica sendo a única a apresentar taxa constante na estação seca para a classe Arachnida, que foi presente em todas as coletas.

$\mathrm{Na}$ tabela 4, verifica-se que as estações climáticas não apresentaram diferenças significativas para os três ambientes analisados e as quatro classes encontradas no estudo. Portanto, a distribuição de indivíduos foi semelhante tanto no período seco quanto no chuvoso.

Analisando os ambientes estudados (Tabela 4), a cultura da macaxeira apresentou maior desuniformidade em relação aos organismos edáficos, com esse fator podendo ser atribuído à ação antrópica, pois os organismos da fauna edáfica são sensíveis às operações de preparo e cultivo do solo (BARETTA et al., 2006). Já na mata atlântica a classe Arachnida teve predominância entre as demais. 
TABELA 4. Variação de organismos por classes de indivíduos da mesofauna em diferentes ambientes em duas estações climáticas.

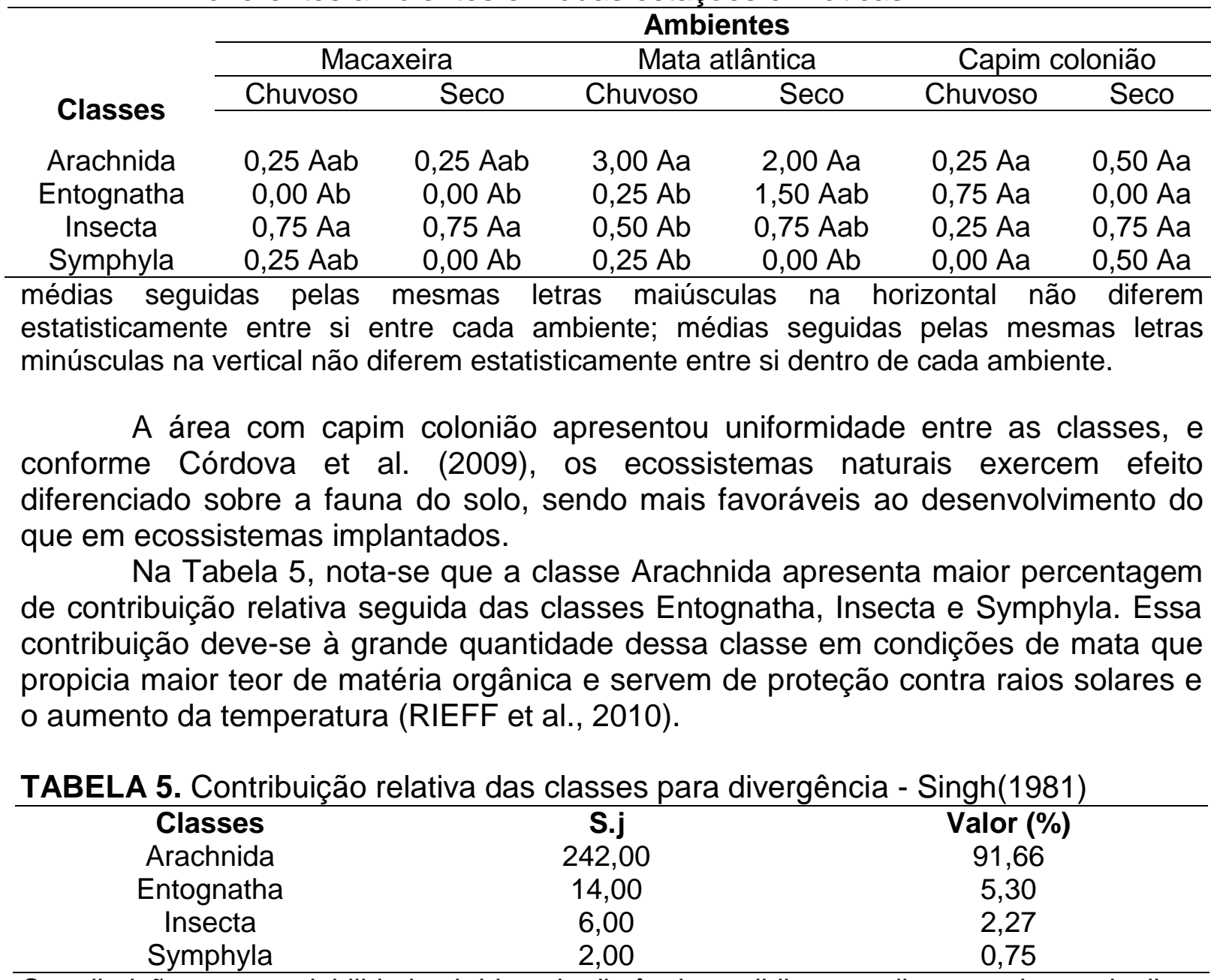

Contribuições para variabilidade obtida pela distância euclidiana media entre classes i a i'.

$\mathrm{Na}$ tabela 6 verifica-se que o maior valor do índice de Shannon ocorreu na estação seca em condições de mata atlântica. Mesmo com o menor número de organismos encontrados nessa estação do que no período chuvoso, a maior diversidade se ocorreu devido a menor dominância de grupos em detrimento de outros. Já o menor valor do índice de Shannon ocorreu na estação seca em área de cultivo de macaxeira.

TABELA 6. Índice de Shannon (h) encontrados nos meses de amostragem da estação chuvosa e estação seca.

\begin{tabular}{ccc}
\hline Ambientes & Estação chuvosa & Estação seca \\
Macaxeira & 0,41 & 0,24 \\
Mata atlântica & 0,36 & 0,51 \\
Macaxeira & 0,41 & 0,47 \\
\hline
\end{tabular}

Considerando o índice Shannon nas estações, as áreas de cultivo de macaxeira e capim no período chuvoso obtiveram valores de 0,41 , enquanto em condições de mata atlântica o referido índice foi apenas 0,51 como está representado na Tabela 6 . Os resultados mostram que o índice de Shannon está relacionado não apenas com o número de indivíduos totais no ambiente, mas

AGRARIAN ACADEMY, Centro Científico Conhecer - Goiânia, v.5, n.9; p.396 2018 
também com a uniformidade das classes dentro do ambiente, tendo em vista que o ambiente de Mata Atlântica apresentou 16 indivíduos, ao contrário dos cultivos de macaxeira e capim colonião que apresentaram apenas cinco cada.

Diferente do que ocorreu na estação chuvosa, na estação seca a mesofauna da mata apresentou maior índice de Shannon que os demais ambientes. Isso porque além de apresentarem maior número de indivíduos, também foi observada maior uniformidade das classes. Na Tabela 7 nota-se que a única correlação positiva que apresentou diferença significativa a $1 \%$ foi o tratamento mata atlântica e o capim colonião.

A maior semelhança entre as condições do solo do capim colonião e a mata atlântica deve-se ao fato da cobertura vegetal permanente e a ausência de revolvimento condicionarem várias características, como maior retenção de umidade, menor amplitude térmica, aumento em agregação, teor de matéria orgânica e ciclagem de nutrientes. Tais fatores são relacionados com índices de maior abundância e diversidade de fauna do solo (BARETTA et al., 2006; ROVEDDER et al., 2009).

TABELA 7. Correlações entre ambientes considerando as classes de indivíduos da mesofauna do solo.

\begin{tabular}{cccc}
\hline Ambientes & Correlação & Probabilidade (\%) & $\mathrm{r} 1(1 \%)$ \\
Macaxeira X Mata atlântica & -1 & 100 & ++ \\
Macaxeira X Capim colonião & -1 & 100 & ++ \\
Mata atlântica X Capim colonião & 1 & 100 & ++ \\
\hline++ Significativo a 1\% de probabilidade pelo teste de Mantel baseado em & 5000 & simulações
\end{tabular}

\section{CONCLUSÕES}

As classes presentes na mesofauna dos três ambientes foram a Arachnida, Entognatha, Insecta e Symphyla.

O ambiente de mata atlântica apresentou maior densidade de organismos da mesofauna;

A classe Arachnida foi classificada como o componente principal com maior poder de diferenciação entre os ambientes.

\section{REFERÊNCIAS}

ALVARES, C. A.; STAPE, J. L.; SENTELHAS, P. C.; GONÇALVES, J. L. M.; SPAROVEK, G. Koöppen's climate classification map for Brazil. Meteorologische Zeitschrift, Berlim, v. 22, n. 6, p. 711-728, 2013.

AGUIAR, W.M.; GAGLIANONE, M. C. Euglossine bee communities in small forest fragments of the Atlantic Forest, Rio de Janeiro state, southeastern Brazil (Hymenoptera, Apidae). Revista Brasileira de Entomologia, Curitiba, v. 56, n. 02, p. 210-219, 2012.

ARAÚJO FILHO, J. C.; BURGOS, N.; LOPES, O. F.; SILVA, F. H. B. B.; MEDEIROS, L. A. R. et al. Levantamento de reconhecimento de baixa e média intensidade dos solos do Estado de Pernambuco. Rio de Janeiro: Embrapa Solos, 2000. 378 
p. (Embrapa Solos. Boletim de Pesquisa, 11). Disponível em: <http://www.cnps.embrapa.br. acesso em: 11 de julho de 2018.

BARETTA, D.; MAFRA, Á.L.; SANTOS, J.C.P.; AMARANTE, C.V.T. \& BERTOL, I. Análise multivariada da fauna edáfica em diferentes sistemas de preparo e cultivo do solo. Pesquisa Agropecuária Brasileira, Brasília, v.41, n.11, p.1675-1679, 2006.

BEGON, M.; HAPER, J. L.; TOWNSED, C. R. Ecology: individuals, populations and communities. 3. ed. Oxford: Blackwell Science, 1996.

BERUDE, M. C.; GALOTE, J. K. B.; PINTO, P. H.; AMARAL, A. A. A mesofauna do solo e sua importância como bioindicadora. Enciclopédia Biosfera, Goiânia, v. 11, n. 22, p. 14-28, 2015.

CÓRDOVA, M; CHAVES, C. L; MANFREDI-COIMBRA, S. Fauna do solo $x$ vegetação: estudo comparativo da diversidade edáfica em áreas de vegetação nativa e povoamentos de pinus SP. Geoambiente On-line, Jatai, v. 1, n. 12, p. 01 $12,2009$.

CALONEGO, J. C.; SANTOS, C. H.; TIRITAN, C. S.; CUNHA JÚNIOR, J. R. Estoques de carbono e propriedades físicas de solos submetidos a diferentes sistemas de manejo. Revista Caatinga, Mossoró, v. 25, n. 2, p.128-135, 2012.

COSTA, E. M.; SILVA, H. F.; RIBEIRO, P. R. A. Matéria orgânica do solo e o seu papel na manutenção e produtividade dos sistemas agrícolas. Enciclopédia Biosfera, Goiânia, v.9, n.17; p. 1842-1860, 2013.

DONAGEMA, G.K.; CAMPOS, D.V.B. de; CALDERANO, S.B.; TEIXEIRA, W.G.; VIANA, J.H.M. Manual de métodos de análise de solos. 2.ed. rev. Rio de Janeiro: Embrapa Solos, 2011. 230p. (Embrapa Solos. Documentos, 132).

MACHADO, D. L.; FERNANDES, C. M. E. R.; DINIZ, A. R.; MENEZES, C. E. G. Fauna Edáfica Na Dinâmica Sucessional Da Mata Atlântica Em Floresta Estacional Semidecidual Na Bacia Do Rio Paraíba Do Sul - RJ. Ciência Florestal, Santa Maria, v. 25, n. 1, p. 91-106, 2015.

NUNES, L. A. P. L.; SILVA, D. I. B.; ADEMIR SÉRGIO FERREIRA DE ARAÚJO, LEITE, L. F. C.; CORREIA, M. E. F. Caracterização da fauna edáfica em sistemas de manejo para produção de forragens no Estado do Piauí. Revista Ciência Agronômica, Fortaleza, v. 43, n. 1, p. 30-37, 2012.

OLIVEIRA, D.; LIMA, R. P.; ERNST. E.; VERBURG, J. Qualidade física do solo sob diferentes sistemas de manejo e aplicação de dejeto líquido suíno. Revista Brasileira de Engenharia Agrícola e Ambiental, Campina Grande, v. 19, n. 3, p. 280-285, 2015.

RIEFF, G. G.; MACHADO, R. G.; STROSCHEIN, M. R. D.; SÁ, E. L. S. Diversidade de famílias de ácaros e colêmbolos edáficos em cultivo de eucalipto e áreas nativas. Revista Brasileira Agrociência, Pelotas, v.16, n.1, p.57-61, 2010. 
RIBEIRO, G. T.; ZANUNCIO, J.C.; TAVARES, W. S.; RAMALHO, F. S.; SERRÃO, J.E. Constancy, Distribution, and Frequency of Lepidoptera Defoliators of Eucalyptus grandis and Eucalyptus urophylla (Myrtaceae) in Four Brazilian Regions. Neotropical Entomology, v. 45, n. 01, p. 629-636, 2016.

ROVEDDER, A.P.M.; ELTZ, F.L.F.; DRESCHER, M.S.; SCHENATO, R.B. \& ANTONIOLLI, Z.I. Organismos edáficos como bioindicadores da recuperação de solos degradados por arenização no Bioma Pampa. Ciência Rural, Santa Maria, v.39, n. 4, p. 1061-1068, 2009.

SCORIZA, R, N.; CORREIA, M, E, F.; ESPINDOLA, J. A. A.; ARAUJO, E, S. Efeito do cultivo de plantas de cobertura sobre a fauna edáfica. Revista brasileira de agroecologia, capão do leão, v. 11, n. 04, p. 310-318, 2016.

SILVA, J.; JUCKSCH, I.; FERES, C. I. M. A.; TAVARES, R. de C. J. Fauna do solo em sistemas de manejo com café. Journal of Biotechnology and Biodiversity, Gurupi, v. 3, n. 2, p. 59-71, 2012. 